\title{
Eficacia del uso de la antibióticoterapia profiláctica en operación cesárea en el Hospital Universitario Metropolitano
}

\author{
Daniel Fajardo Arrieta*; Guillermo Acosta Osio**; Antonio Rojano Tejeda***
}

RESUMEN: Se estudiaron 45 pacientes del 21 de diciembre de 1990 al 15 de julio de 1991, con embarazo con producto viable, y fueron sometidas a cesárea urgente y programada en el Departamento de Ginecología y Obstetricia.

De manera aleatoria a 15 pacientes (Grupo A) se les administró ampicilina, a 15 pacientes (Grupo B) se les administró cefradina, ambas drogas se aplicaron perioperatoriamente como profilaxis; a las 15 restantes (Grupo C) no se le administró ninguna droga.

Hallándose que en los grupos A y B no se encontró morbilidad infecciosa y en el Grupo C la morbilidad infecciosa fue de un $26.7 \%$.

Se concluye que la antibióticoterapia profiláctica en nuestro medio disminuye un $100 \%$ la morbilidad infecciosa.

PALABRAS CLAVES: Antibióticoterapia profiláctica, Operación cesárea, Morbilidad infecciosa.

SUMMARY: From december 21, 1990 thru july of 1991, forty five patients were studied and observed. Such patients were pregnant and about ready to deliver and had to be rushed and subdued to have an emergency cesarean operation, and programmed in the Department of Ginecology and Obstetrics.

At random, we provided 15 patients (group A) With Ampicilin, another set of 15 patients (group B) were give Cefradina, both drugs were applied priorly as prophylaxis; the last 15 patients, (group C) were not given any drug. found.

It was found that in group A or Group B no infectious Morbidity was given with in group C a rate of $26.7 \%$ of infectious morbidity was

It is concluded that in our community the prophylactic antibiotictherapy lowers and minimizes a 100\% any type of infectious morbidity.

KEY WORDS: Prophylactic antibiotic-therapy, Cesarean operation, Infectious morbidity.

\section{Introducción}

La operación cesárea es la cirugía más común en la práctica ginecoobstétrica diaria. El reciente aumento de la tasa de cesárea ha dado como resultado un notable aumento de las infecciones puerperales. Las infecciones puerperales pueden manifestarse simplemente por síndrome febril, considerando que la fiebre durante el puerperio es un signo más común después del parto abdominal que del vaginal. También puede manifestarse como endometritis, la cual es más frecuente y grave después de la cesárea que el parto vaginal $(6$, 13).

En los últimos 20 años se han realizado incontables estudios perfectamente planeados acerca de la profilaxis

\footnotetext{
* Residente III Año Ginecología y Obstetricia, Hospital Universitario Metropolitano.

** Profesor y Director Dpto. Ginecología y Obstetricia, Hospital Universitario Metropolitano.

*** Coordinador Postgrado Ginecología y Obstetricia, Universidad Metropolitana.
}

antibiótica para la cesárea y los resultados de estas investigaciones permiten sacar conclusiones informales.

Se han usado múltiples antibióticos profilácticos especialmente los de amplio espectro, solos o combinados, sin embargo se ha demostrado que la combinación aumenta la toxicidad y no aumenta la efectividad. El antibiótico ideal sería uno con amplio espectro de acción que cubra variedad de gérmenes aerobios y anaerobios, grampositivos y gramnegativos que tengan al mismo tiempo baja toxicidad, buena concentración en los tejidos y fácil administración (19).

En Colombia son pocos los trabajos publicados referentes a la profilaxis antibiótica en operación cesárea, y en Barranquilla concretamente no se ha publicado ninguna investigación al respecto. Situación que ha motivado a investigar la eficacia de los antibióticos (ampicilina y cefradina) en la profilaxis de la cesárea.

En nuestro medio, el índice de infección puerperal posterior a una cesárea es relativamente alto, esto puede deberse a que en algunas instituciones no se tenga en cuenta los factores de riesgo que se presentan y no se lleve a cabo una adecuada 
asepsia y antisepsia. Los resultados del trabajo permiten observar la eficacia de la utilización de los antibióticos (ampicilina y cefradina) y tenerlos en cuenta como medio de control a las infecciones puerperales posterior a una cesárea en una forma corta en cuanto a tiempo y a la vez económica.

El trabajo podrá mostrar la utilidad clínica de la profilaxis antibiótica en la operación cesárea en relación con la morbilidad infecciosa materna durante el puerperio.

Además permite clasificar los factores de riesgo existentes en nuestro medio; identificar las complicaciones postoperatorias que se presentan en nuestro servicio como: síndrome febril, infección de herida quirúrgica, absceso de pared, absceso de pared más endometritis, hematoma más infección de herida quirúrgica, dehiscencia de histerorrafia más infección. Comparar los resultados teniendo en cuenta el tipo de antibiótico recibido o utilizado y la recepción o no del tratamiento.

La hipótesis general de este trabajo consiste en demostrar que la morbilidad infecciosa a que está expuesta una mujer embarazada que será sometida a cesárea se puede disminuir con el uso de antibióticos profilácticos. Teniendo en cuenta para ello que existen ciertos factores de riesgo como son: las horas de trabajo de parto, si se presenta o no ruptura de membrana, el tiempo quirúrgico, estado de hemoglobina de la paciente y edad gestacional de embarazo.

Como hipótesis específica trata de demostrar que la antibióticoterapia profiláctica en cesárea puede evitar las complicaciones infecciosas en el postoperatorio en un $100 \%$.

Los factores de riesgo de nuestro servicio que se tuvieron en cuenta para realizar el presente estudio fueron: la edad gestacional, número de tactos vaginales, tiempo quirúrgico, el tipo de cirujano, la indicación de la cesárea, tipo de incisión en abdomen y útero, la hemoglobina de ingreso y hemoglobina postoperatoria, número de horas de trabajo de parto y número de horas de ruptura de membranas.

Se presentan limitaciones que anoto a continuación:

No se ha demostrado en nuestro medio cual es el germen que se presenta como causa más frecuente de infección puerperal, no se cuenta con un adecuado aporte económico por lo cual el grupo de pacientes en estudio es un poco limitado.

\section{Marco teórico}

Reseña histórica. El punto clave en la evolución de la cesárea se produjo en 1882 cuando Max Sanger, introdujo las suturas en la pared uterina, basándose en estudios de Ohio y Lousiana, en los cuales habían realizado suturas con hilo de plata (1).

Antes de las recomendaciones de Max Sanger sobre la sutura de la herida uterina, Eduardo Porro propuso en 1876, su técnica de operación cesárea-histerectomía con lo que se logró reducir la mortalidad materna de $50 \%$ a $15 \%$. La eficacia de la operación de Porro se debía a dos factores: en primer lugar con esta técnica se eliminaba el cuerpo uterino y sus anexos infectados y, en segundo, la operación facilitaba el drenaje externo por medio de un procedimiento de eventración que fijaba el muñón cervical a la pared abdominal anterior (1). Durante los 70 años siguientes, se hicieron varias modificaciones a la operación de Porro para luchar contra la amenaza de septicemia postoperatoria.
Así, en 1945 se llegó a recomendar la extirpación del útero como la operación más segura para casos de infección (2).

Otro de los métodos para evitar la contaminación era la operación de exclusión que aislaba la zona prevista para la incisión de la cavidad peritoneal antes de abrir el útero. Las primeras operaciones de este tipo fueron efectuadas por Frank y Sellheim, pero ambos vieron malogrados sus esfuerzos por perfeccionar un procedimiento extraperitoneal. Las operaciones por exclusión fueron utilizadas por poco tiempo y abandonadas luego porque la línea de cierre peritoneal resultaba ineficaz para evitar la filtración de los loquios (1).

La posibilidad de parto extraperitoneal fue sugerida por primera vez por Joerg. En EE. UU. la posibilidad de realizar una verdadera operación extraperitoneal fue sugerida en 1824 por Phillip Physick. Pero no fue hasta 1907 cuando un afamado ginecólogo alemán, Fritz Frank, intentó realizar la idea de Physick. Un año más tarde en 1908, la misma vía fue utilizada por Hugo Sellheim (1).

En 1908 Wilheim Latzko (3), médico vienés, ideó una operación más sencilla utilizando la vía paravesical. En los años siguientes en EE. UU. se producen modificaciones de la técnica extraperitoneal de Latzko, como Aldridge, Irwin y Norton $(1,3,4)$.

La primera cesárea realizada en Colombia tuvo lugar en el Departamento de Antioquia en 1844, el cirujano fue el doctor José Ignacio Quevedo, no anotó el tipo de cesárea pero tanto la madre como el niño sobrevivieron (14).

Técnicas operatorias.La cesárea puede ser intraperitoneal y extraperitoneal:

La cesárea intraperitoneal puede ser de tipo corporal o clásica, tipo Beck (segmento-corporal), tipo Kerr (segmentaria) (5).

La cesárea extraperitoneal puede ser paravesical, supravesical y la combinada $(1,4)$.

Frecuencia. Durante los últimos veinte años en todos los hospitales de todas las regiones geográficas ha habido un aumento espectacular en el índice de los partos por cesárea que varió en menos de 5\% en 1965 a más del 30\% en 1988 (6).

En el Hospital Universitario Metropolitano el porcentaje total de cesárea en el año 1987 fue de $22.7 \%$ (7).

Farmacología y la embarazada. Hay pocos medicamentos de uso común cuyo efecto es independiente de la dosis prescrita o, de manera más directa, de la concentración de sus moléculas en sangre o en el órgano blanco. Generalmente, los factores fisiológicos y patológicos ejercen una influencia mucho mayor sobre la concentración de los medicamentos que sobre la capacidad de respuesta de los órganos blancos, y el embarazo no es una excepción. En teoría, la absorción, distribución, metabolismo y excresión del medicamento, denominados en el conjunto variables "Fármaco-cinéticas", pueden estar influidas por cambios fisiológicos que ocurren durante el embarazo (8).

Absorción de los medicamentos. El estómago se vacía lentamente y se retrasa la motilidad intestinal (influida por concentraciones elevadas de progesterona); por tanto, el tránsito gastrointestinal se prolonga en 30 a 50\%, esto puede reducir la velocidad y la integridad de la absorción medicamentosa. El vaciado gástrico lento retrasa la respuesta a los medicamentos y se prefiere la vía parenteral para su administración (8). 
Distribución de los medicamentos. La distribución de las moléculas a través del organismo depende de su solubilidad en los lípidos (que determina la transferencia a través de las membranas lípidas y su captación por el tejido adiposo) y su inclinación a fijarse a proteínas tanto en el plasma como en el tejido extravascular. Una vez que se ha realizado la distribución el volumen aparente de ésta es la constante proporcional relacionada con la cantidad del medicamento en el organismo y la concentración de éste medida en el plasma. Esto no se relaciona con órganos o comportamiento de líquidos corporales. Las sustancias con volúmenes de distribución aparentemente pequeños tienen solubilidad lípida baja y se fijan fuertemente a las proteínas plasmáticas, mientras que aquellas con volúmenes grandes de distribución son sumamente solubles en lípidos y con frecuencia se fijan fuertemente a las proteínas tisulares. Rara vez el volumen real tiene consecuencias tanto para el paciente como para el médico a menos que cambie inesperadamente (8).

Durante el embarazo el contenido total de agua en el organismo aumenta hasta 8 litros ( $80 \%$ es extracelular) y el volumen del plasma se incrementa en un $50 \%$. La concentración de albúmina en el plasma cae 5-10 gr/l, y los ligandos endógenos, como los ácidos grasos libres, compiten con los medicamentos por los sitios de fijación tanto de la albúmina como la glucoproteína alfa 1 ácida.

Un aumento en el volumen de distribución reducirá el nivel máximo de concentración del medicamento después de una dosis y retardará su eliminación, a menos que haya un aumento simultáneo en la depuración por metabolismo o excresión (8).

La consecuencia de una reducción de la fijación en las proteínas del plasma son, a primera vista, sorprendentes. La mayor fijación, por cualquier razón, produce una fracción mayor de medicamento no fijo: estas moléculas son libres para escapar de la circulación, distribuirse en forma más amplia, ejercer efectos farmacológicos y ser eliminadas por metabolismo o excresión. Durante el embarazo, el resultado neto del incremento gradual en la fracción no fija es una concentración total del medicamento más baja en el plasma, siendo la concentración del medicamento no fijo semejante a la que ocurre cuando no hay embarazo (8).

Metabolismo de los medicamentos. Los medicamentos que son suficientemente hidrosolubles para escapar de la resorción del túbulo renal, después de la filtración glomerular, se eliminan en forma inalterada. Los medicamentos liposolubles deben volverse más polares mediante metabolismo, antes de su excreción por la bilis o la orina; esto se logra comúnmente por medio de oxidación (por ejemplo hidroxilación, desmetilación) o por la conjugación (glucorónido o sulfato).

La actividad de las enzimas que metabolizan medicamentos se refleja por la excreción urinaria de productos endógenos de desecho, como 6-beta hidroxicortisol y ácido D-glucárico, que son índices indirectos útiles. Durante el embarazo, el aumento en la excreción de estos productos y las pruebas histológicas de hiperplasia del retículo endoplásmico de superficie lisa en las células hepáticas indican que el metabolismo del medicamento es estimulado (inducido) y se considera que la progesterona es el agente causal en gran parte; si esta inducción origina un aumento mensurable en la depuración por el hígado, la eliminación se acelerará y se acortará la semidesintegración (9).

Aunque también se produce matabolismo de medicamentos tanto en la placenta como en el hígado fetal, se considera pequeña la contribución relativa de estos órganos en la depuración de los medicamentos del organismo materno (8).

Excreción del medicamento. Durante el embarazo, el flujo plasmático renal aumenta hasta casi duplicarse y hay incrementos sustanciales tanto en el índice de filtración glomerular (70\%) como en la depuración de creatinina: la depuración de los medicamentos que se excretan sin modificarse (como la gentamicina y digoxina) es directamente proporcional a la depuración de la creatinina (10).

Sensibilidad de los receptores. No hay pruebas de que los receptores maternos ola sensibilidad tisular a los medicamentos se alteren durante el embarazo.

En resumen, las variables farmacocinéticas que se aceptan predeciblemente por los cambios fisiológicos relacionados con el embarazo son el volumen aparente de distribución, la fijación a las proteínas del plasma, la actividad enzimática metabolizante y la depuración renal (8).

Agentes antimicrobianos. La depuración de ampicilina, la cual se excreta sin alterarse en grado considerable a través del riñón, es más rápida en la mayoría de las etapas del embarazo, pero su semidesintegración sólo es un poco más corta porque el volumen de distribución aparente aumenta en casi 50\%; por tal razón la concentración máxima que se logra después de cada dosis es reducida, lo mismo que su vida media, principalmente por el mayor volumen de distribución y la dosis convencionales deben duplicarse cuando se trata de infecciones generales, para infección de vías urinarias no se requieren cambios en la dosificación (11).

Aunque la depuración y la semidesintegración de la cefuroxima se altera en la misma dirección de la ampicilina, la magnitud del cambio es menor; no obstante, a causa del aumento de la depuración (no hay cambio en el volumen de distribución), las concentraciones plasmáticas son menores después de las dosis recomendadas y éstas últimas deben aumentarse (11-12).

La ampicilina y cefradina, son dos antimicrobianos que inhiben la síntesis de mucopéptido en la pared bacteriana, conduciendo a la producción de formas con pared deficiente, que se lisan en un medio ambiente osmóticamente protegido, siendo por lo tanto bactericidas, y debido a que actúan frente a estructuras únicas en las bacterias, generalmente no es tóxico para las células del huésped (11-12).

Frecuencia de la infección en cesárea. La fiebre (con temperatura mayor o igual a $38^{\circ} \mathrm{C}$ ) durante el puerperio, es un signo más común después del parto abdominal que después del parto vaginal. Su resolución suele ser espontánea después del parto vaginal, pero generalmente persiste después de la cesárea (13).

En un estudio de 1000 mujeres grávidas, Filker y Monif observaron que en $65(6.5 \%)$ la temperatura subió a más de $38^{\circ} \mathrm{C}$ durante las primeras 24 horas después del parto. La mitad de estas pacientes (33) tuvieron un parto vaginal y representaban el $9.8 \%$ de todos los partos vaginales. En el $80 \%$ (26) de estas 33 pacientes se observó resolución espontánea de la fiebre. Las otras 32 fueron sometidas a cesárea y representaban el $22.5 \%$ de todos los partos abdominales. En 
este grupo, la fiebre cedió espontáneamente sólo en el 30\% (9) pacientes. La etiología en esta fiebre no ha sido aclarada, aunque muchos autores consideran que podría deberse a la deshidratación o bien ser una reacción al paso de las proteínas fetales. También es posible que algunas de estas fiebres sean manifestación de una infección uterina leve y de una curación espontánea (13).

La endometritis (diagnosticada con base en criterios clínicos) es más frecuente y grave después de cesárea que después del parto vaginal. Después del parto abdominal, la frecuencia de endometritis suele fluctuar entre 12 y $95 \%$, mientras que después del vaginal raramente pasa del $3 \%$. En términos generales, el riesgo es de 5 a 10 veces superior después de cesárea. La gravedad de la infección puede medirse tanto por el fracaso de un tratamiento con antibióticos como por las complicaciones graves que ocurren más a menudo en mujeres con partos abdominales (15); así mismo, se ha observado más casos de muerte por septicemia después de la cesárea.

La mayor frecuencia de infección en la cesárea podría explicarse por varios factores, como manipulación intrauterina más considerable, reacciones a cuerpos extraños (sutura), necrosis tisular a nivel de la línea de sutura, formación de hematoma o seroma, infección de la herida y parto difícil y prolongado. Después de la cesárea, la fuente más común de infección es el útero, cuya infección recibe diferentes nombres: endometritis, endomiometritis, endoparametritis o simplemente metritis. Aunque los signos para diagnosticar endometritis varían generalmente incluyen fiebre, hipersensibilidad del útero, loquios pupulentos o fétidos, leucocitosis periférica y ausencia de otros sitios de infección. Síntomas y signos no específicos como malestar, dolor abdominal, escalofríos y taquicardia son también muy frecuentes. Sin embargo, muchas veces el diagnóstico es presuntivo, porque la mayor parte de las pacientes no presentan el cuadro clínico completo. En casi todos los casos de infección uterina, los primeros signos y síntomas aparecen durante los cinco primeros días después de la cesárea $(6,13)$.

Factores de riesgo. Es evidente que el riesgo no es el mismo para todos los pacientes que serán sometidos a una cesárea. Así, aquellos con operaciones programadas de manera electiva (sin trabajo de parto y sin rotura de las membranas) tendrán índices más bajos de infección que con procedimientos no electivos $(6,13)$.

En cuanto al trabajo de parto D Angelo y Sokol, utilizando el análisis diferencial para eliminar las variables que causan confusión, encontraron que el factor más importante relacionado con la morbilidad puerperal después de una cesárea era la duración del trabajo. Otros estudios, empleando técnicas estadísticas similares, han confirmado este resultado (23).

Comola contaminación bacteriana de la cavidad amniótica es frecuente después de la rotura prematura de membrana, resulta lógico pensar que la rotura prematura de membrana desempeña un papel importante en la infección postcesárea. Gilstrop y Cunnigham (13) señalan en su estudio que la totalidad de las 56 muestras de líquido amniótico recogidas en el momento de la operación cesárea de mujeres con rotura de membrana de mayor de 6 horas contenían microorganismos, el $95 \%$ de estas mujeres tuvieron endometritis.
En México se han diseñado estudios para determinar la flora bacteriana cervicovaginal en mujeres sanas, grávidas, no grávidas y puerperas, encontrando un elevado porcentaje de bacterias potencialmente patógenas tales como enterobacterias, staphylococcus aureus y bacteroides observando además que existen diferencias en el patrón bacteriano normal inducido por el embarazo y el puerperio. Así mismo se ha tratado de establecer, cuáles son los gérmenes predominantes aislados con mayor frecuencia en rotura de membrana, llegando a la conclusión de que es staphylococcus epidermis (6).

En dos estudios recientes el número de exámenes vaginales está correlacionado con el peligro de infección, sin embargo, en otros estudios esta variable clínica no fue identificada como factor de riesgo (13).

Independiente de la raza, los pacientes indigentes presentan índice más elevado de infección puerperal que los de clase media (13).

Los resultados de varios estudios mostraron una relación entre la anemia y las infecciones puerperales. La obesidad no ha sido un factor de riesgo consistente para la infección genital, aunque sí lo es para la infección de las heridas en cirugía general (13).

Microbiología clínica. En las muestras de cavidad uterina, los microorganismos aerobios se encuentran aproximadamente en el $70 \%$ de las pacientes con endometritis. A menudo los bacilos gramnegativos forman la mayor parte de los cultivos para aerobios. En este grupo, Escherichia Coli es el más frecuente (hasta en un $30 \%$ de las pacientes), seguido por Kleibsiella y Proteus mirabilis en aproximadamente el 5\% de las pacientes, en tanto que enterobacter, pseudomonas y otras especies ocurren con menor frecuencia. Aerobios grampositivos, de los cuales los estreptococos son los más frecuentes también han sido aislados en muchos pacientes. Los estreptococos del grupo B aparecen en $15 \%$ de los cultivos genitales de pacientes en endometritis.

En cambio los estreptococos del grupo A, sólo se encuentran en menos del $1 \%$ de estos pacientes. Los enterococos en subgrupo de los estreptococos del grupo D incluye streptococcus fecalis y streptococcus faecium y han sido identificados muchas veces en cultivos endometriales.

En pacientes que fueron tratadas con antibióticos profilácticos (cefalosporinas), los enterococos suelen encontrarse en el $25 \%$ de los cultivos. Nisseria gonorrhoeae, un coco gramnegativo facultativo, se encuentra raramente por lo general, forma parte de una infección mixta (13).

Los microorganismos anaeróbicos desempeñan un papel netamente más importante en las infecciones puerperales y se encuentran en $80 \%$ de los cultivos genitales de mujeres con endometritis recogidos y manejados correctamente. Los microorganismos aislados con más frecuencia son miembros del género bacteroides; estos bacilos gramnegativos son importantes debido a su participación en la formación de abscesos intraperitoneales y a su resistencia a muchos antibióticos. Aunque en la última década del grupo B. Fragilis ha atraído la atención de varios autores, el B. Bivius es el anaerobio que con más frecuencia se ha aislado del tracto genital en muchos estudios recientes. Tanto B. Fragilis como B. Bivius son resistentes a muchos antibióticos. 
Otros anaerobios aislados a menudo son los estreptococos anaerobios (especie peptococcus y petostreptococcus) y las especies fusobacterium y clostridium. Por lo general, estos organismos son sensibles a los antibióticos más utilizados (13).

También se han encontrado miooplasmas genitales (mycoplasma-hominis y Ureplasma urealiticum) en la circulación sanguínea de mujeres febriles durante el puerperio (16).

Wagner y col.(13) encontraron una relación entre cultivos cervicales positivos para Chlamidia trachomatis durante el preparto y a la aparición tardía de endometritis moderada después del parto vaginal.

Profilaxis antibiótica para la cesárea. Se define como el uso de antibióticos en aquella situación en la cual no existe evidencia de proceso infeccioso alguno y se pretende evitarlo mediante su utilización (17).

Desde 1968 se han reportado la eficacia de varios estudios acerca del uso de antibióticos profilácticos en la cesárea, Miller y Crichton reportaron desde esa fecha que la profilaxis con ampicilina fue efectiva en la disminución de la infección postoperatoria. Se ha incrementado la lista encontrándose la penicilina, carbenicilina, ticarcilina, piperacilina, cefalotina, cefalodina, cefazolina, cefamandole, cefoxtin, cefradina, cefoperazona, cefotaxime, moxalactam, cefotetan, canamicina, gentamicina, clindamicina, metronidazol y sulfametoxazol-trimetoprim se han usado en profilaxis quirúrgica y han demostrado eficacia (18).

Se han usado múltiples antibióticos profilácticos especialmente los de amplio espectro, solos o combinados; sin embargo, se ha demostrado que la combinación aumenta la toxicidad y no aumenta la efectividad (19).

Se ha demostrado que casi todos los regímenes profilácticos causan disminución de la endometritis en un $75 \%$ (20). Gran parte de la reducción en las infecciones suelen atribuirse a disminuciones en las infecciones uterinas y de la herida.

Algunos estudios han comparado la eficacia de los regímenes profilácticos. En las comparaciones de ampicilina con cefalotina, cefalotina con cefamandole, y penicilina con clindamicina más gentamicina, no se encontraron diferencias significativas desde el punto de vista estadístico en los índices de infección (13).

Hoy día, la mortalidad por la operación cesárea es prácticamente nula, pero la morbilidad infecciosa observada por diversas instituciones, en los casos en los cuales no se utilizó antibiótico-profilaxis, era entre 20 a $50 \%$, en cambio, en aquellos casos en que sí se utilizó algún tipo de antibióticoprofilaxis, la morbilidad infecciosa se encontró entre 10 a $20 \%$ además, se observó que la morbilidad infecciosa también se veía afectada según el tipo de institución, el tipo de pacientes y la calidad de la asistencia prenatal, entre otros factores (21).

Después de comparar los resultados obtenidos con períodos cortos y largos de profilaxis, los autores han favorecido los cursos cortos. D Angelo y col. (23), compararon regímenes profilácticos con cefalosporinas, de 24 horas y cinco días y no encontraron diferencias significativas en el índice de infección postoperatoria (29\% en régimen corto contra $20 \%$ en el de cinco días). En una revisión de 26 estudios Swartz y Grolle (23), calcularon que los regímenes de 12 horas o menos son igualmente eficaces comparándolos con cursos profilácticos más largos.

En estudios comparados de profilaxis antes y después de la ligadura del cordón umbilical, Gordon y col., encontraron índices parecidos de infección postoperatoria con ampicilina profiláctica antes y después de la ligadura del cordón (13).

En otros estudios se compararon los resultados de profilaxis de dosis única de 2 dosis y de 3 dosis, y no encontraron diferencias importantes en elíndice de infección postoperatoria en esos estudios; pero los tres regímenes redujeron de manera significativa las infecciones en comparación con el grupo de placebo (13).

En el patrón profilaxis antibiótica con tres dosis, se ha observado una excelente influencia en la flora vaginal. Las cefalosporinas y especialmente la cefazolina y cefoxitin se le ha observado selección por E. faecalis. En un estudio hecho por Faro y col. demostraron que solamente 2 gramos de cefazolina, 2 gramos de ampicilina, 4 gramos de piperacilina y 1 gramo de cefotetan fueron más efectivos que otros antibióticos en la reducción de la incidencia de endometritis postparto (22).

\section{Materiales y métodos}

Se realizó un estudio prospectivo, comparativo y experimental desde el 21 de diciembre de 1990 al 15 de julio de 1991. Se incluyeron 45 pacientes divididos en 3 grupos en forma aleatoria: 15 pacientes recibieron ampicilina (Grupo A), 15 pacientes recibieron cefradina (Grupo B), y 15 pacientes no recibieron antibiótico (Grupo $\mathrm{C}$ ).

En el estudio se aplicaron los siguientes criterios de inclusión:

- Embarazo con producto viable.

- Sometidas a operación cesárea por cualquier indicación urgente o programada .

Los criterios de exclusión fueron:

- Temperatura a su ingreso igual o mayor que $37.5^{\circ} \mathrm{C}$.

- Datos de corioamnionitis.

- Alérgica al medicamento utilizado.

- Antecedente de antibióticoterapia por cualquier razón por lo menos dos semanas previas a la cesárea.

- Datos de proceso infeccioso concomitante a cualquier otro nivel.

Se designó previamente el esquema que cada paciente recibiría, a los que les correspondieron el Grupo A se le administró ampicilina dos gramos intravenosamente, hasta completar un total de 3 dosis, la primera dosis aplicada al momento del pinzamiento del cordón umbilical y luego cada 4 horas.

Las pacientes asignadas al Grupo B se les administró cefradina 1 gramo intravenosamente hasta completar un total de 3 dosis, la primera dosis aplicada al momento del pinzamiento del cordón umbilical y luego cada 4 horas, bajo la supervisión del observador.

Las pacientes que fueron asignadas al Grupo C (Grupo testigo) no recibieron antibiótico.

A su ingreso se les solicitó cuadro hemático completo y examen general de orina. Durante su estancia hospitalaria se les solicitó nuevo cuadro hemático completo a las 48 horas del puerperio quirúrgico y fueron vigiladas en forma estrecha sus constantes vitales y la evolución de su puerperio, como 
involución uterina, características de la herida y los loquios así como la sintomatología para poder descartar proceso infeccioso a cualquier otro nivel.

Se utilizó un protocolo para obtener la información, que se elaboró, en el cual registra datos acerca de información personal, antecedentes personales, ginecoobstétricos, tipo de procedimiento operatorio, terapéuticos, tipo de cirujano, complicaciones quirúrgicas, control postoperatorio inmediato y a los 7 días.

El análisis estadístico de la información obtenida se hizo utilizando técnicas descriptivas e inferenciales para lo cual se hicieron tablas univariables y multivariables. Para establecer correlación de variables y probar la hipótesis se usaron las pruebas de Fisher s: $\mathrm{X}^{2}$ (Chi-cuadrado) y t de student con un nivel de confianza del $95 \%$.

\section{Resultados y discusión}

En el presente trabajo se estudiaron 45 pacientes embarazadas desde el 21 de diciembre de 1990 al 15 de julio de 1991 . Se dividieron en 3 grupos: El Grupo A estuvo formado por 15 pacientes a las cuales se les administró ampicilina; el Grupo B estuvo formado por 15 pacientes a las cuales se les administró cefradina; y el Grupo C estuvo formado por 15 pacientes a las cuales se les administró antibiótico.

La información se consignó en un protocolo, previamente elaborado, la cual se procesó y se obtuvo el siguiente resultado.

El grupo de pacientes presentó una edad entre los 20 y 39 años, siendo homogénea en los 3 grupos; lo mismo puede decirse de la distribución según la paridad y peso, factores que no influyen en la producción de morbilidad infecciosa en este estudio.

En cuanto a la edad gestacional una paciente del Grupo A y otra del Grupo B presentaron una edad gestacional de 35 semanas, siendo 41 semanas de edad gestacional la mayor presentada y estuvo distribuida en los 3 grupos de pacientes en forma homogénea (Tabla 1).

Tabla 1

DISTRIBUCION DE LAS CARACTERISTICAS GENERALES DE LA POBLACION ESTUDIADA SEGUN GRUPOS

\begin{tabular}{|c|c|c|c|c|c|}
\hline \multirow[b]{2}{*}{ Características } & Grupo & \multicolumn{2}{|c|}{ Grupo } & \multicolumn{2}{|c|}{ Grupo } \\
\hline & A $\%$ & & $\%$ & $\mathrm{C}$ & $\%$ \\
\hline \multicolumn{6}{|l|}{ Edad } \\
\hline $20-25$ & $1173.4 \%$ & 8 & $53.3 \%$ & 5 & $33.3 \%$ \\
\hline $26-30$ & $213.3 \%$ & 6 & $40.0 \%$ & 7 & $46.7 \%$ \\
\hline$>30$ & $213.3 \%$ & 1 & $6.7 \%$ & 3 & $20.0 \%$ \\
\hline Total & $15100 \%$ & 15 & $100 \%$ & 15 & $100 \%$ \\
\hline \multirow[t]{2}{*}{ Promedio de edad } & \multirow{2}{*}{$\begin{array}{l}\bar{x} \cdot \text { D.E. } \\
25.8 \pm 4.8\end{array}$} & \multirow{2}{*}{\multicolumn{2}{|c|}{$\begin{array}{l}\bar{x} \text { D.E. } \\
25.6 \pm 3.3\end{array}$}} & \multirow{2}{*}{\multicolumn{2}{|c|}{$\begin{array}{l}\bar{x} \text { D.E. NS } \\
27.5 \pm 5.2\end{array}$}} \\
\hline & & & & & \\
\hline \multicolumn{6}{|l|}{ Paridad } \\
\hline $0-3$ & $15 \quad 100 \%$ & 14 & $93.3 \%$ & 13 & $86.7 \%$ \\
\hline$>3$ & - & 1 & $6.7 \%$ & 2 & $13.3 \%$ \\
\hline Total & $15 \quad 100 \%$ & 15 & $100 \%$ & 15 & $100 \%$ \\
\hline
\end{tabular}

\begin{tabular}{lrrrrrr}
$\begin{array}{l}\text { Peso } \\
60-65 \mathrm{~K}\end{array}$ & 2 & $13.3 \%$ & 9 & $60 \%$ & 6 & $40.0 \%$ \\
$66-75 \mathrm{~K}$ & 11 & $73.4 \%$ & 4 & $26.7 \%$ & 7 & $46.7 \%$ \\
$>75 \mathrm{~K}$ & 2 & $13.3 \%$ & 2 & $13.3 \%$ & 2 & $13.3 \%$ \\
\cline { 2 - 7 } & 15 & $100 \%$ & 15 & $100 \%$ & 15 & $100 \%$ \\
Total & & & & & & \\
Edad gestacional & & & & & & \\
$<37$ semanas & 3 & $20.0 \%$ & 1 & $6.7 \%$ & 3 & $20.0 \%$ \\
$>37$ semanas & 12 & $80.0 \%$ & 14 & $93.3 \%$ & 12 & $80.0 \%$ \\
Total & 15 & $100 \%$ & 15 & $100 \%$ & 15 & $100 \%$ \\
& & & & & &
\end{tabular}

En cuanto a la hemoglobina preoperatoria no hubo diferencia significativa en los promedios de los 3 grupos, así como tampoco en la hemoglobina postoperatoria (Tabla 2), por lo cual esto no constituyó un factor de riesgo en este estudio por cuanto los niveles estaban dentro de los parámetros normales (mayor de $10 \mathrm{gr} \%$ ) en promedio, aunque se presentó un caso con niveles inferiores que se analiza posteriormente. Nielson T.F. y Ott W.J. (1983) encontraron relación entre la anemia y las infecciones puerperales.

Referente al número de horas del trabajo de parto DAngelo y Sokol (1980) encontraron que el factor más importante relacionado con la morbilidad puerperal después de una cesárea era la duración del trabajo, en el presente estudio el número de horas del trabajo de parto previos a la cesárea tuvo una variación de 2 a 21 horas, siendo semejante los promedios para los 3 grupos (Tablas 2 y 3). Se encontró que en el Grupo C el cual no recibió antibiótico, la duración en los promedios del trabajo de parto fue menor que los 2 grupos que lo recibieron, $\mathrm{y}$ a pesar de esto hubo morbilidad infecciosa en el Grupo C (Tablas 2, 3, y 7).

Tabla 2

\section{ANTIBIOTICOTERAPIA PROFILACTICA EN OPERACION CESAREA. CARACTERISTICAS GENERALES DE LOS GRUPOS}

\begin{tabular}{|c|c|c|c|}
\hline & Grupo A & Grupo B & Grupo C \\
\hline Características & $\bar{X}$ DE. & $\overline{\mathrm{X}}$ DE. & $\overline{\mathrm{X}}$ DE. \\
\hline Hb.Preoperatoria & $12.6 \pm 1.2$ & $12.4 \pm 1.1$ & $12.8 \pm 0.98$ NS \\
\hline $\begin{array}{l}\text { Hb.Postoperatoria } \\
\text { Disminución } \mathrm{Hb} \text {. }\end{array}$ & $11.48 \pm 1.5$ & $11.3 \pm 1.2$ & $11.9 \pm 1.2 \quad$ NS \\
\hline $\begin{array}{l}\text { Postoperatoria } \\
\mathrm{N}^{\circ} \text { Horas de }\end{array}$ & 1.2 & 1.1 & 0.9 \\
\hline $\begin{array}{l}\text { Parto } \\
\text { Tiempo Quirúrgico }\end{array}$ & $2.8 \pm 4.3$ & $4.8 \pm 6.2$ & $0.93 \pm 1.48 \mathrm{NS}$ \\
\hline (minutos) & $49 \pm 7.1$ & $34.3 \pm 5.6$ & $40 \pm 9.2 \mathrm{NS}$ \\
\hline
\end{tabular}

Tabla 3

DISTRIBUCION DE FRECUENCIA DE HORAS DE TRABAJO DE PARTO SEGUN ESQUEMA O GRUPO

\begin{tabular}{|c|c|c|c|c|c|}
\hline \multirow{2}{*}{$\begin{array}{l}\text { T. Parto Esquema } \\
\text { (horas) }\end{array}$} & \multirow{2}{*}{$\begin{array}{l}\text { Grupo A. } \\
\mathrm{N}^{\circ} \%\end{array}$} & \multicolumn{2}{|c|}{ Grupo B. } & \multicolumn{2}{|c|}{ Grupo C. } \\
\hline & & $\mathrm{N}^{\circ}$ & $\%$ & $\mathrm{~N}^{\circ}$ & $\%$ \\
\hline $0-10$ & 1493.3 & 13 & 86.6 & 15 & 100 \\
\hline $11-20$ & 6.7 & 1 & 6.7 & & - \\
\hline$>-20$ & - & 1 & 6.7 & & - \\
\hline Total & \begin{tabular}{|l|l|}
15 & 100
\end{tabular} & 15 & 100 & 15 & 100 \\
\hline
\end{tabular}


En cuanto al tiempo quirúrgico no fue significativo porque a pesar de que en el grupo $\mathrm{A}$ el tiempo promedio fue mayor y en el Grupo B el tiempo promedio fue menor, no presentaron morbilidad infecciosa (Tabla 2).

Jiménez B.E. (1986), observó una tendencia a infectarse más cuando las pacientes se operan más rápido. De las 45 cesáreas 2 fueron practicadas por ginecólogos y el resto por médicos residentes en formación.

Tabla 4

TIPO INCISION EN LA PARED ABDOMINAL

\begin{tabular}{|c|c|c|c|c|c|c|}
\hline \multirow[b]{2}{*}{ Tipo } & \multicolumn{2}{|c|}{ Grupo A } & \multicolumn{2}{|c|}{ Grupo B } & \multicolumn{2}{|c|}{ Grupo C } \\
\hline & $\mathrm{N}^{\circ}$ & $\%$ & $\mathrm{~N}^{\circ}$ & $\%$ & $\mathrm{~N}^{\circ}$ & $\%$ \\
\hline Pfannenstiel & 5 & 33.3 & 1 & 6.7 & 3 & 20 \\
\hline Mediana & 10 & 66.7 & 14 & 93.3 & 12 & 80 \\
\hline Total & 15 & 100 & 15 & 100 & 15 & 100 \\
\hline
\end{tabular}

Con relación al tipo de incisión en pared abdominal se encontró que las dos pacientes que presentaron absceso de pared, a una se le practicó incisión mediana y a la otra incisión tipo pfannenstiel no siendo significativo para demostrar que la incisión transversal presenta mayor morbilidad infecciosa (Tabla 4). El tipo de incisión en el útero fue para todas las cesáreas tipo Kerr.

Tabla 5

\section{DISTRIBUCION SEGUN INDICACION DE LA CESAREA POR} GRUPOS

\begin{tabular}{|c|c|c|c|c|}
\hline $\begin{array}{l}\text { Indicación } \\
\text { de la } \\
\text { cesárea }\end{array}$ & Grupo A & Grupo B & Grupo C & Total \\
\hline $\begin{array}{l}\text { Desproporción } \\
\text { cefalopélvica. } \\
\text { Presentación }\end{array}$ & n & 2 & 3 & 10 \\
\hline $\begin{array}{l}\text { pélvica } \\
\text { Cesárea }\end{array}$ & 2 & 3 & 4 & 9 \\
\hline $\begin{array}{l}\text { anterior } \\
\text { Embarazo }\end{array}$ & 1 & 4 & 2 & 7 \\
\hline gemelar & 1 & 2 & 1 & 4 \\
\hline Otras & 6 & 4 & 5 & 15 \\
\hline Total & 15 & 15 & & \\
\hline
\end{tabular}

Las indicaciones de cesárea fueron semejantes en los 3 grupos, siendo la más frecuente por desproporción cefalopélvica, presentación pélvica, cesárea anterior y embarazo gemelar. Dentro del grupo de otros se encontró toxemia severa, sufrimiento fetal agudo, placenta previa, várices vulvares y feto macrosómico (Tabla 5). Gibbs R.S. (1980, 1985 ) reporta que las pacientes que serán sometidas a cesárea programada de manera electiva tendrán índices más bajos de infección que aquellas con cirugía de urgencia. De las 4 pacientes que presentaron morbilidad en el estudio, 3 fueron cesáreas electivas y una de urgencia, lo que demuestra que no hubo relación con el estudio anteriormente citado.
Tabla 6

ESTADOS DE LAS MEMBRANAS

\begin{tabular}{|c|c|c|c|c|c|c|}
\hline \multirow{2}{*}{$\begin{array}{l}\text { Estado de las } \\
\text { Membranas }\end{array}$} & \multicolumn{2}{|c|}{ Grupo A } & \multicolumn{2}{|c|}{ Grupo B } & \multicolumn{2}{|c|}{ Grupo C } \\
\hline & $\mathrm{N}^{\circ}$ & $\%$ & $\mathrm{~N}^{\circ}$ & $\%$ & $\mathrm{~N}^{\circ}$ & $\%$ \\
\hline Integras & 13 & 86.7 & 13 & 86.7 & 14 & 93.3 \\
\hline Rotas & 2 & 13.3 & 2 & 13.3 & 1 & 6.7 \\
\hline Total & 15 & 100 & 15 & 100 & 15 & 100 \\
\hline
\end{tabular}

Se encontró en el Grupo A dos pacientes (13.3\%) con ruptura de membrana; en el Grupo B dos pacientes (13.3\%) con ruptura y una paciente (6.7\%) en el Grupo C. Gilstrap, Cunningham y Blanco citados por Gibss (1985) relaciona la ruptura de membrana con una morbilidad infecciosa de un 95\%. Aquí observamos (Tabla 6) que las cuatro pacientes de los Grupos A y B a pesar de tener ruptura de membrana no presentaron morbilidad y que la paciente del Grupo $C$ presentó morbilidad infecciosa (endometritis), por lo que observábamos que se previno completamente la morbilidad infecciosa en pacientes con ruptura de membranas.

Tabla 7

\section{DISTRIBUCION DE LA MORBILIDAD SEGUN ESQUEMA TERAPEUTICO O GRUPO}

\begin{tabular}{|c|c|c|c|c|c|c|}
\hline \multirow[b]{2}{*}{ Infección } & \multicolumn{2}{|c|}{ Si } & \multicolumn{2}{|c|}{ No } & \multicolumn{2}{|c|}{ Total } \\
\hline & $\mathrm{N}^{\circ}$ & $\%$ & $\mathrm{~N}^{\circ}$ & $\%$ & $\mathrm{~N}^{\circ}$ & $\%$ \\
\hline Grupo A & - & - & 15 & 100 & 15 & 100 \\
\hline Grupo B & - & - & 15 & 100 & 15 & 100 \\
\hline Grupo C & 4 & 26.7 & 11 & 73.3 & 15 & 100 \\
\hline$X^{2}=6.38$ & & & & & & \\
\hline
\end{tabular}

En cuanto a la morbilidad según el esquema terapéutico se encontró que en los grupos A y B que recibieron antibióticos profilácticos no presentaron morbilidad infecciosa, o sea que la respuesta fue de un $100 \%$, lo cual es significativo comparado con otros estudios como el de Larry C.F. y col. (1987) en donde habla de una respuesta de un 77 a un $98 \%$ según el antibiótico que utilice, Miller RD (1968), Harger JH (1981) y Gibbs RS y col. (1972) reportan que en estudios efectuados administrando antibióticos profilácticos la morbilidad infecciosa fue de 10 a $20 \%$ dependiendo de los factores de riesgo. En el grupo C $26.7 \%$ (4 pacientes) presentaron riesgo directo a exposición, siendo el $\mathrm{X}^{2}=6.38$ y la $\mathrm{t}=\mathrm{p}<0.05$ (Tabla 7), siendo estadísticamente significativo, ya que los autores mencionados encontraron una morbilidad infecciosa en los grupos que no utilizaron antibióticos profilácticos de un 10 a $85 \%$. 
Tabla 8

\section{DISTRIBUCION SEGUN ESQUEMA TERAPEUTICO Y MORBILIDAD}

\begin{tabular}{|c|c|c|c|c|c|c|}
\hline \multirow[b]{2}{*}{ Tipo de morbilidad } & \multicolumn{2}{|c|}{ Grupo A } & \multicolumn{2}{|c|}{ Grupo B } & \multicolumn{2}{|c|}{ Grupo C } \\
\hline & $\mathrm{N}^{\circ}$ & $\%$ & $\mathrm{~N}^{\circ}$ & $\%$ & $\mathrm{~N}^{\circ}$ & $\%$ \\
\hline Absceso de pared & - & - & - & - & 2 & 50 \\
\hline Síndrome febril & - & - & - & - & 1 & 25 \\
\hline Endometritis & - & - & - & - & 1 & 25 \\
\hline Total & - & - & - & - & 4 & 100 \\
\hline
\end{tabular}

En el grupo de las 4 pacientes infectadas se presentó 2 casos de absceso de pared, 1 caso de endometritis y 1 caso de síndrome febril; siendo el tipo de infección más frecuente en las cesáreas del servicio el absceso de pared con un $50 \%$ (Tabla 8).

Esto difiere con estudios realizados por Walls Rodríguez (1990) que encuentra como causa de mayor morbilidad postoperatoria el síndrome febril.

\section{Tabla 9}

\section{DISTRIBUCION DE LA FRECUENCIA DEL GRUPO QUE NO RECIBIO ANTIBIOTICO EN QUE HUBO INFECTADAS EN RELACION CON LA EDAD GESTACIONAL}

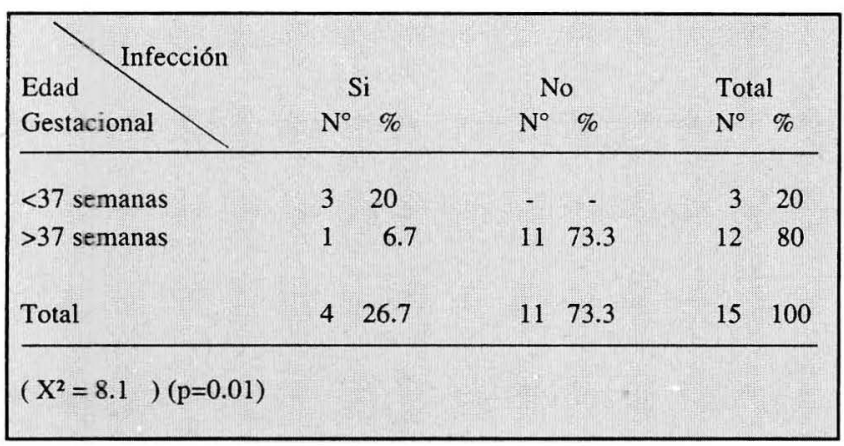

Con relación al grupo que no recibió antibiótico se encontróque el $20 \%$ ( 3 de las pacientes) presentaron edad gestacional igual o menor de 37 semanas, lo que nos indica que hay mayor riesgo de infección en las pacientes que se someten a cesárea con edad gestacional menor de 37 semanas, siendo estadísticamente significativo ya que se encontró el $\mathrm{X}^{2}=8.1$ y la $\mathrm{p}=0.01$ (Tabla 9). Teniendo en cuenta que una paciente (6.7\%) presentó edad gestacional mayor a 37 semanas (39 semanas) y se infectó, nos llama la atención que la hemoglobina de esta paciente pasó de $12.2 \mathrm{gr} \%$ en el preoperatorio a $9.7 \%$ en el postoperatorio, siendo posiblemente la causa de su morbilidad infecciosa, pero no es significativo estadísticamente. La morbilidad infecciosa se puede prevenir en las pacientes con edad gestacional menor de 37 semanas con antibióticoterapia profiláctica, ya que en el grupo A se presentaron 3 pacientes (20\%) y en el grupo B se presentó una paciente $(6.7 \%)$ con edad gestacional menor o igual a 37 semanas y estas no presentaron signos de infección.

\section{Conclusiones}

La utilización de la ampicilina a razón de 2 gr y de la cefradina a razón de $1 \mathrm{gr}$. en forma profiláctica al pinzar el cordón umbilical por 3 dosis cada 4 horas previno la aparición de morbilidad infecciosa en un $100 \%$, en el postoperatorio de nuestras pacientes.

A menor edad gestacional del embarazo la paciente presenta mayor tendencia a la morbilidad infecciosa con una $p=0.01$, la cual se puede prevenir en nuestro medio con la ampicilina y cefradina.

Se demostró que un régimen corto de antibióticoterapia profiláctica de 3 dosis por vía endovenosa es eficaz y económico en nuestro medio.

Se demostró que la administración del antibiótico en el momento de pinzar el cordón fue electiva, y con esto evitamos la exposición del niño al antibiótico, así como enmascarar una infección neonatal.

No se presentó ningún efecto desfavorable como hipersensibilidad a la ampicilina o cefradina en este estudio, pero se debe tener en cuenta en el momento de administrar los antibióticos.

\section{Recomendaciones}

A raíz de este trabajo se recomienda establecer el uso de cualquiera de los dos esquemas de profilaxis antibiótica en pacientes que serán sometidas a cesárea con factores de riesgo, en el Hospital Universitario Metropolitano y en las otras instituciones de nuestro medio que se quieran acoger a él.

\section{BIBLIOGRAFIA}

1. Hibbard Lester T. Cesárea extraperitoneal. Clínicas Obstétricas y ginecológicas. Editorial Interamericana. Madrid, España. 1985. Vol 4

2. Cosgrove S.A. An Evaluation of extraperitoneal cesarean section. Am. J. Obstet. Gynecol. 1946; 52: 237.

3. Latzko W. Der Extraperitoneale Kaiserachnitt Seine Geschichte, seine technik and seine indikationen wien klin wochnschr. 1909; 22: 477.

4. Norton JF. Paravesical extraperitoneal cesarean section technique. Am. J. Obstet. Gynecol. 1946; 51: 519.

5. Acosta Bendek E. y col. Manual de normas de obstetricia y ginecología. Hospital Universitario Metropolitano. Editorial Calendario Espriellabe y asoc. Ltda. Barranquilla.1987.
6. Walss RR., Esparza M. Antibióticoterapia profiláctica en operación cesárea. Ginec. obstet. México. 1990; 58-78-82.

7. Acosta Osio G. Cesárea en el Hospital Universitario Metropolitano. Revista Unimetro. 1988; 4 (8): 22-27.

8. Mucklow JC. Destino de los medicamentos durante el embarazo. Ginecología y Obstetricia, temas actuales. España. 1986; 13: 2.

9. Frantz AG., Katz IH. y Jailer JW. 6-Betahidroxicortisol. High Levels in human urine in pregnancy and toxemia. Procedings of the society for experimental biology and medicine. 1960; 105: 41-43.

10. Davinson JM. The urinary sistem clinical physiology in obstetrics. Oxford: Blackwell scientific 1980; 289-327. 
11. Phillipson A. Pharmacokinetics of ampicilin during pregnancy. Journal of infections diseases. 1977; 136: 297-309.

12. Phillipson A. y Striernstedt G. Pharmacokinetics of cefuroxime in pregnancy. Am. Journal of obstetrics and ginecology. 1982; 142: 823 828.

13. Gibbs RS. Infección después de la cesárea. Clínicas obstétricas y ginecológicas. Editorial Interamericana. España 1985; (4): 877.

14. Sánchez Torres F. Historia gineco-obstétrica en Colombia. Revista Colombiana de Obstetricia y Ginecología. 1988; Vol. XXXIX, № 2.

15. Gibbs RS., Jones PM., Wilder CJ. Antibiotico Terapy of endometritis following cesarean section. Obst. Gynecol. 1978; 52: 31.

16. Wallace RJJr., Alpert S., Browmek, Lin JSL., Mc Cormack WM., Kenney GE. Mycoplasma hominis from blood cultures in patients with postpartum fever. Obstet. Gynecol. 1978; 51: 181.

17. Goodman GA., Goodman SL., Rall WT., Murad F. Farmacología. 7a. edición. Editorial Médica Panamericana. 1985; 1019.
18. Galask RP. Chaging concepts in obstetric antibiotic prophylaxis Am. J. Obstet. Gynecol. 1987; 157: 2.

19. Jiménez BE. Profilaxis de infección en cesárea con mefoxitin. Revista Colombiana de Obstetricia y Ginecología. 1986; Vol. XXXVII $\mathrm{N}^{\circ} 1$.

20. Ehrenkranz J., Blacwelder WC., Pfaft SJ., Poppe D., RRA. Yerg DE. MA. MSPH, Kaslow RA. Infections complicating low-risk cesarean sections in comunity hospitals efficacy of antimicrobial prophylaxis. Am. J. Obstet. Gynecol. 1990; 162: 2.

21. Faro S., Martens MG., Hammill HA., Riddle G., Tortolero G. Antibiotic prophylaxis: is there a difference? Am. J. Obstet. Gynecol. 1990; 162: 4.

22. Escobedo L. JM. et al. Antibiotics prophylactic-in cesarean section. Gynecol.-Obstet. México 1991; 59: 35-38.

23. D Angelo LJ., Sokol RJ. Short-Versus long-course prophylactic antibiotic treatment in cesarean section patients. Obstet. Gynecol. 1982; 145: 158.

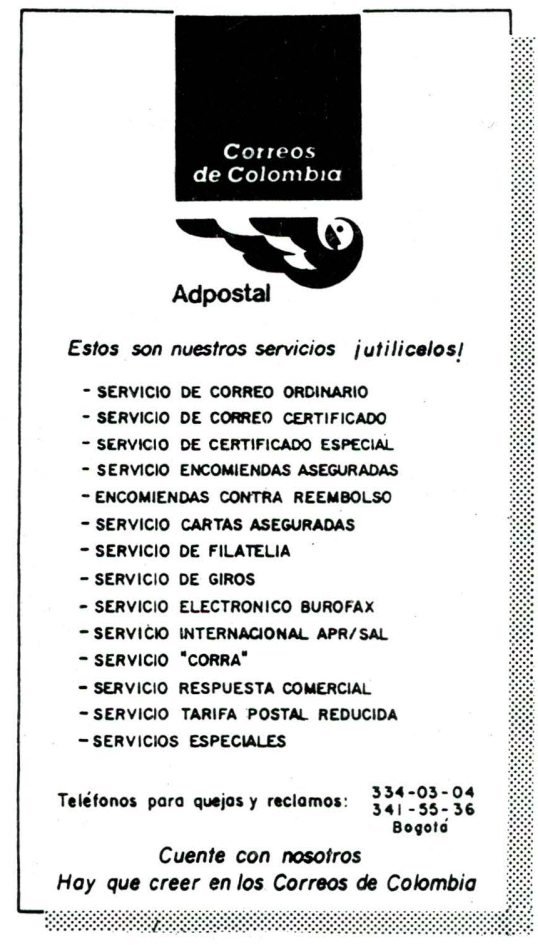

